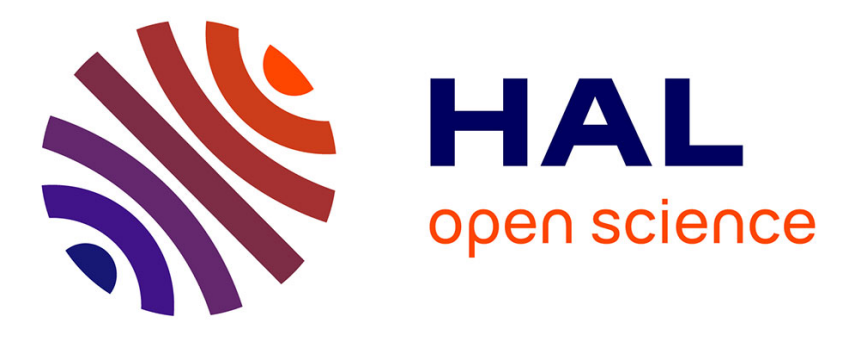

\title{
Hydro mechanical behaviour of a heterogeneous swelling clay material
}

\author{
Christophe Imbert, Claude Gatabin, Pascal Maugis, David Leboulch, \\ Emmanuel Mouche
}

\section{- To cite this version:}

Christophe Imbert, Claude Gatabin, Pascal Maugis, David Leboulch, Emmanuel Mouche. Hydro mechanical behaviour of a heterogeneous swelling clay material. Poromechanics II: Proceedings of the Second Biot Conference on Poromechanics, Aug 2002, Grenoble, France. hal-02939258

\section{HAL Id: hal-02939258 \\ https://hal.science/hal-02939258}

Submitted on 15 Sep 2020

HAL is a multi-disciplinary open access archive for the deposit and dissemination of scientific research documents, whether they are published or not. The documents may come from teaching and research institutions in France or abroad, or from public or private research centers.
L'archive ouverte pluridisciplinaire HAL, est destinée au dépôt et à la diffusion de documents scientifiques de niveau recherche, publiés ou non, émanant des établissements d'enseignement et de recherche français ou étrangers, des laboratoires publics ou privés. 


\title{
Hydro mechanical behaviour of a heterogeneous swelling clay material
}

\author{
C. Imbert \& C. Gatabin, \\ P. Maugis*, D. Leboulch*, E. Mouche*, \\ Commissariat à l'Energie Atomique (CEA), DPC/SCCME, DM2S/SFME*, SACLAY, France.
}

\begin{abstract}
This paper presents the main results of a research program devoted to the experimental study and the modelling of the hydro mechanical behaviour of an heterogeneous swelling clay material. This material is constituted of a mixture of bentonite powder and high dry density bentonite pellets. It may be used to seal shafts and galleries thanks to its low permeability and large swelling capacity. The behaviour of the material during the hydration phase, observed experimentally on small scale samples, is compared to numerical simulations.
\end{abstract}

\section{INTRODUCTION}

An important item for the long term safety of High Level Waste underground repository is the backfilling and sealing of access shafts and connection galleries. Bentonite is widely studied as backfill and sealing material in geological repositories for radioactive waste because of its low permeability and large swelling capacity. The use of precompacted high density blocks is generally proposed. CEA has developed, in cooperation with other European Research institutions, SCK.CEN in Belgium and CIEMAT in Spain, an industrial scale technique to produce a granular backfill material that is composed of bentonite powder mixed with high dry density bentonite pellets. To demonstrate the applicability of this type of material on an industrial scale, it has been applied for the sealing of the "experimental shaft" of the HADES Underground Research Laboratory at Mol in Belgium. The study of the hydromechanical behaviour of this large scale seal and of small scale samples in laboratory constitutes the main task of the European project RESEAL (REpository SEALing, in Volckaert 2000 and Dereeper 2001).

The objectives of the surface laboratory characterization consist in understanding the behaviour of the heterogeneous bentonite material and in providing to modellers the main hydromechanical properties in order to get the parameters of the constitutive laws. The mixture is initially unsaturated, so when hydrated under confining conditions the mixture generates a swelling pressure due to hydro-mechanical coupling. As well as for highly compacted bentonite blocks, it is necessary to determine the water resaturation kinetics, the swelling pressure increase, and the water permeability after complete saturation. These properties are related to the dry density of the mixture, this parameter being a key parameter in the study. This paper presents the results obtained on small scale samples.

\section{DESCRIPTION OF THE EXPERIMENTAL PROGRAM}

\subsection{Characteristics of the heterogeneous swelling clay material.}

Different techniques to make pellets or aggregates have been developed and intensively tested by CEA (Volckaert 1996 \& 2000). Among these different processes, this paper focused on the particular technique developed during the BACCHUS and RESEAL european projects, that is a dynamic compaction between two rotating wheels. This industrial scale technique has been applied to different bentonites to product high density pellets : French bentonite FoCa coming from the Paris Basin, and Spanish bentonite Serrata extracted near Almeria. The specific behaviour of each bentonite during the compaction must be taken into account, the process being very sensitive to many parameters, particularly the initial water content of the clay powder. The pellets exhibit high dry density (table 1) and a good cohesion.

The mixture giving the best global dry density is composed of $70 \%$ of pellets and $30 \%$ of powder (proportion in dry mass). But a more homogeneous hydration is obtained with a mixture composed of 
$50 \%$ of pellets and $50 \%$ of powder. The material has been mainly studied with this 1:1 composition.

Table 1 : Dry density of the pellets.

\begin{tabular}{ccc}
\hline Clay & $\begin{array}{c}\text { Massic water content } \\
(\%)\end{array}$ & Dry density $\left(\mathrm{g} / \mathrm{cm}^{3}\right)$ \\
\hline FoCa & 7.7 & 2.00 \\
FoCa & 14.4 & 1.86 \\
Serrata & 9.2 & 1.89 \\
Serrata & 10.3 & 1.87 \\
Serrata & 13.8 & 1.87 \\
\hline
\end{tabular}

\subsection{Experimental device}

The device is constituted of an oedometric cell made of stainless steel, with an inside diameter of 120 $\mathrm{mm}$, especially designed to work with the mixture (fig. 1). It is suited to the size of a pellet (approximately $25 \times 25 \times 12 \mathrm{~mm}$ ). The sample of mixture is confined into a rigid cylinder between two pistons. Between each piston and the clay sample, a stainless steel filter allows the exchanges of fluids, air or water between clay and drainage pipes. The cell can receive samples that height is comprised between $30 \mathrm{~mm}$ and $150 \mathrm{~mm}$, and even more using a long cylinder. The cell is placed under a rigid frame equipped with sensors. A load cell provides the total axial load exerted by the clay on the piston. A displacement sensor gives the relative displacement between the two pistons and consequently the volume variation during the test. A computer ensures the data acquisition.

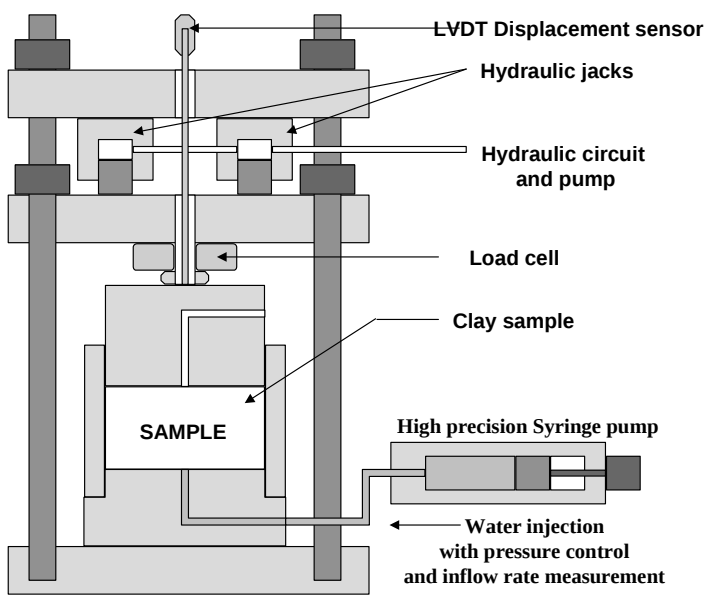

Figure 1. Schematic presentation of the experimental device.

\subsection{Experimental protocol}

The mixture is carefully hand-made from the two components inside the cell in order to obtain an homogeneous repartition of the pellets inside the sample. The dry density is adjusted by compacting axially the clay mixture by slow loading. After unloading, the sample is ready to be confined under the loadframe and to undergo the hydration phase. Its main features consist in its height, its dry density, water content and overconsolidation ratio.
The clay sample is hydrated by applying a water pressure at the bottom face. The air exhaust is allowed at the top. During all the test the water pressure is regulated using a high precision syringe pump, and the volume of water absorbed by the sample is recorded versus time. A distilled and deaired water is used. All conditions lead to an 1D hydration experiment. Due to the rigidity of the load frame, the relative displacement of the confining pistons is very weak (nevertheless it is recorded), and the test can be regarded as a constant volume test.

The axial force exerted by the clay on the piston, divided by the piston area, gives an average value of the swelling pressure (after deduction of the water pressure). At the end of the experiment, the sample is extracted from the cell and cut into slices. Destructive measurements of the water content and bulk density are made to determine the homogeneity of the sample along the hydration axis.

Many tests have been performed on this type of material, but in this paper we focused on a series of tests made on the $50 \%$ pellets, $50 \%$ powder mixture of FoCa and Serrata clays. Two parameters have been particularly studied : the height and the initial dry density of the mixture.

\section{HYDRO-MECHANICAL BEHAVIOUR OF THE PELLETS/POWDER MIXTURE}

\subsection{Behaviour of the clay mixture during the hydration phase}

The objectives of this series of tests are : first to record the increase of swelling pressure and water uptake during hydration, that means observing the kinetics, second to determine the time necessary to reach the complete saturation, and third to measure the swelling pressure developed by the saturated material and also the hydraulic conductivity of it.

Due to a strong hydromechanical coupling, wellknown in the swelling clays, the swelling pressure (determined from the axial force exerted by the clay) is increasing. The evolution of the swelling pressure versus time is not monotonous but exhibit a special shape : after a first maximum, the pressure is decreasing, then stabilising and then a new increase leads to the final value, figure 2 . At the same time, the evolution of water uptake is monotonous. The sample is regarded as saturated when the swelling pressure is constant, and when water uptake is stable or shows a constant slope. Calculations based on the final destructive measurements of bulk density and water content confirm that the material is saturated. This particular behaviour is observed for different dry densities of the samples :1.30, 1.50, 1.60 and 1.65 (fig.3). The mixture made of pellets and powder of Serrata clay shows the same hydromechanical behaviour, figure 4 . 
To explain this evolution of swelling pressure, it was assumed that the powder is hydrating and swelling before the pellets, generating the first peak. But different other experiments demonstrated that this assumption was false. Pasquiou demonstrated that the behaviour is the same when the powder is replaced by non swelling powder such as kaolinite (Pasquiou 2001). Destructive and non destructive measurements on the two phases of the mixture, at the first stages of the hydration, showed that the hydration of powder and pellets is delayed about only a few hours (Pasquiou 2001, Volckaert 2000). As a conclusion, it appears that pellets and powder hydrate quite simultaneously and both contribute to the swelling.

This behaviour cannot be attributed to an insufficient size of the cell, because the same evolution of the swelling pressure is observed when the diameter of the cell is eight times the maximal dimension of the pellet.

The evolutions of the swelling pressure and the water uptake versus time are very different, and no simple relation can be found between these two measured parameters.

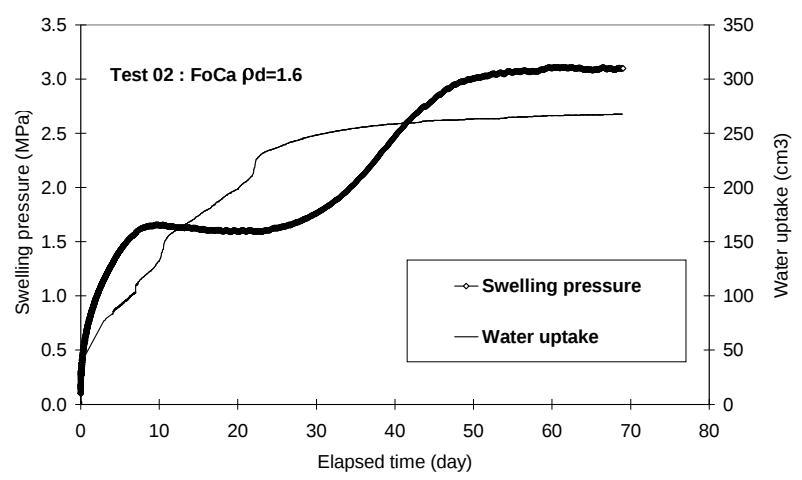

Figure 2. Evolution of the swelling pressure and water uptake versus time, mixture of FoCa clay, dry density $1.6 \mathrm{~g} / \mathrm{cm}^{3}$.

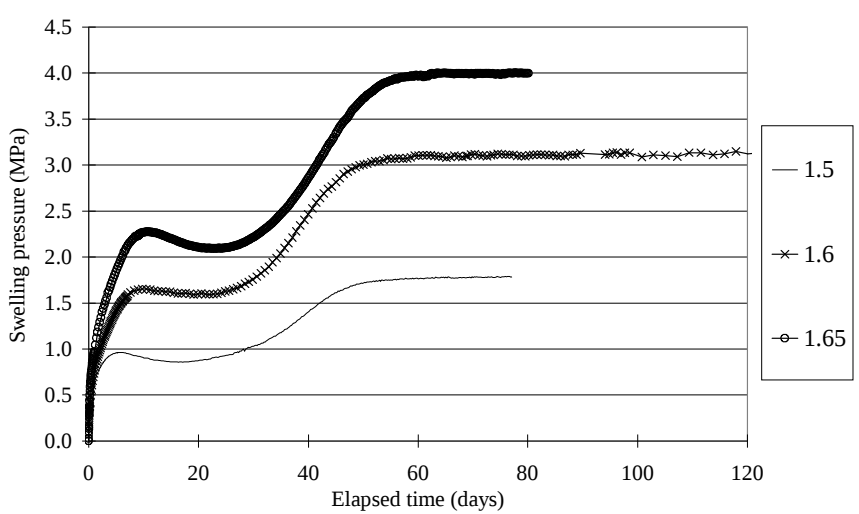

Figure 3. Swelling pressure of mixtures of pellets and powder of FoCa clay, for different dry densities.

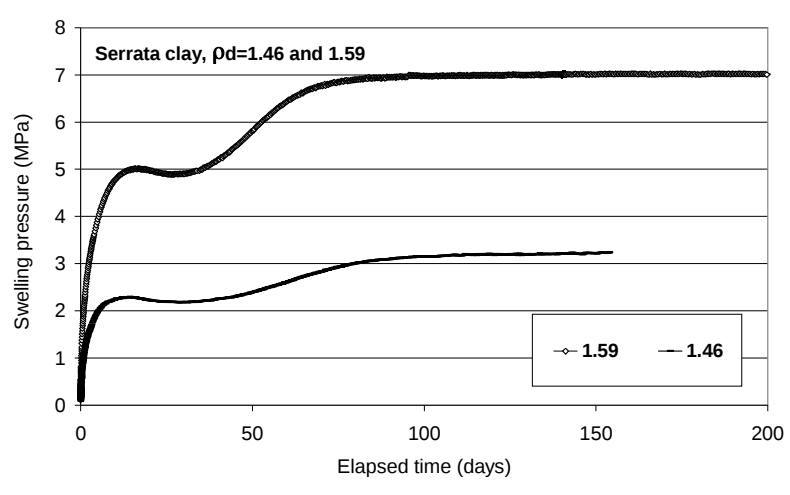

Figure 4. Evolution of the swelling pressure of 2 mixtures of powder and pellets of Serrata clay.

\subsection{Behaviour of the totally saturated clay mixture}

Many measurements have been made to verify if the mixture is either saturated and homogeneous at the end of the test. For the little height samples, whose height is comprised between $30 \mathrm{~mm}$ and $50 \mathrm{~mm}$, final destructive measurements indicate that the clay is saturated and that either the dry density and water contents are quite homogeneous.

The swelling pressure of the mixture of pellets and powder has been compared to the swelling pressure given by the block of compacted clay. For the same dry density, the swelling pressure of the mixture is slightly lower than the swelling pressure provided by the homogeneous compacted clay (fig.5). The behaviour of the compacted clay (blocks) has been established for high dry densities. The difference of swelling pressure can be explained by a little difference between two batches of the same FoCa French clay. A particular relationship between $\rho d$, the dry density of the mixture of pellets and powder, and the swelling pressure Ps obtained at complete saturation is proposed for the two clays :

Ps $=0.0113(\rho d)^{12.29}$ for FoCa clay

Ps $=0.0047(\rho d)^{14.241}$ for Serrata clay

Ps being the swelling pressure expressed in MPa.

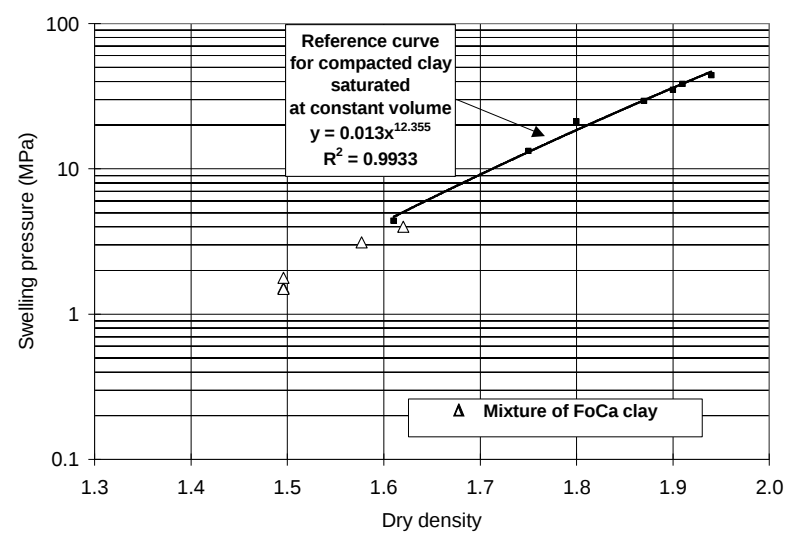

Figure 5. Swelling pressure versus dry density for a block compacted clay and for a mixture of powder and pellets.

\section{MODELING}




\subsection{Model, laws and parameters}

We summarize the hydraulic and mechanical laws and parameters used for the modeling. Only FoCa clay is considered here. The physical parameters were not thoroughly known, especially for density 1.6, which is the one analysed in this paper, so Serrata parameter values were used where FoCa's were unavailable. These data apply to a formerly compacted sample.

The hydro-mechanical model is described by Darcy's law:

$\mathrm{S} \frac{\partial \omega}{\partial \mathrm{t}}+\omega \frac{\partial \mathrm{S}}{\partial \mathrm{t}}+\operatorname{div}\left(-\mathrm{K} \vec{\nabla} \mathrm{P}_{1}\right)=\mathrm{q}$

with $\mathrm{S}$ saturation, $\omega$ porosity, $\mathrm{K}$ permeability, $\mathrm{P}_{1}$ liquid pressure, $\mathrm{q}$ source term. The porosity increment $\mathbf{d} \omega$ is linked to the strain tensor increment $\mathrm{d} \varepsilon$, given by the mechanical model, through the relationship $\mathrm{d} \omega=\operatorname{tr}(\mathrm{d} \varepsilon)$.

The hydraulic properties are given in (Volckaert 2000). The capillary law is a Van Genuchten law, the intrinsic permeability is given by Kozeny's expression, the relative permeability to water follows a cubic law.

The mechanical model is Alonso's model for partially saturated soils (Alonso 1990). It is governed by the following constitutive equations.

The total stress is $\boldsymbol{\sigma}$, the net stress $\boldsymbol{\sigma}_{\text {net }}=\boldsymbol{\sigma}-\mathrm{P}_{\mathrm{g}} \mathbf{I}$, with $\mathrm{P}_{\mathrm{g}}$ the gas pressure, and effective stress $\boldsymbol{\sigma}_{\text {eff }}=\boldsymbol{\sigma}-\mathrm{P}_{\mathbf{l}} \mathbf{I}$. $\boldsymbol{\sigma}^{\prime}=\boldsymbol{\sigma}_{\text {net }}$ if unsaturated, or $\boldsymbol{\sigma}_{\text {eff }}$ if saturated : $\boldsymbol{\sigma}^{\prime}=\boldsymbol{\sigma}-$ $\max \left(\mathrm{P}_{\mathrm{g}}, \mathrm{P}_{\mathrm{l}}\right) \mathbf{I}=\boldsymbol{\sigma}-\mathrm{s} \mathbf{I}+\mathrm{P}_{\mathrm{g}} \mathbf{I}, \mathrm{s}=\mathrm{P}_{\mathrm{g}}-\mathrm{P}_{\mathrm{l}}$ being the suction.

- Elastic deformations :

$\mathrm{d} \varepsilon_{\sigma}^{v, e}=-\frac{\kappa}{1+e} \frac{d p^{\prime}}{p^{\prime}}$

$\mathrm{d} \varepsilon_{s}^{v, e}=-\frac{\kappa_{s}}{1+e} \frac{d s}{s+P_{a t m}}$

Where e : void ratio $=\omega /(1-\omega), P_{a t m}$ : atmospheric pressure.

$\kappa(s)$ : stiffness to p' increase

$=\kappa_{0}\left(1-\alpha_{\kappa} \ln s\right)(\mathrm{s}$ in $\mathrm{MPa})$

$=\kappa_{0}$ when $\mathrm{s} \geq 1$.

$\kappa_{0}=0.054 ; \alpha_{\mathrm{K}}=0.16$

$\kappa_{\mathrm{s}}\left(\mathrm{s}, \mathrm{p}^{\prime}\right)$ : stiffness to $\mathrm{s}$ increase

$=\kappa_{\mathrm{s} 0}\left(1-\alpha_{\mathrm{ks}} \ln \left(\mathrm{p}^{\prime} / \mathrm{p}_{\mathrm{ref}}\right)\right) \mathrm{e}^{-\beta \mathrm{ks} \mathrm{s}}$

$\kappa_{\mathrm{s} 0}=0.3 ; \alpha_{\mathrm{ks}}=0.1638 ; p_{\mathrm{ref}}=0.01 \mathrm{MPa} ; \beta_{\mathrm{Ks}}=0.03 \mathrm{MPa}$

1

- Total plastic deformation :

$\mathrm{d} \varepsilon_{\sigma}^{\mathrm{v}, \mathrm{p}}=-\frac{\lambda-\mathrm{k}}{1+\mathrm{e}} \mathrm{d} \ln \mathrm{P}_{0}=-\frac{\lambda_{0}-\mathrm{K}_{0}}{1+\mathrm{e}} \mathrm{d} \ln \mathrm{P}_{0}^{*}$

$=-\frac{\lambda_{\mathrm{s}}-\mathrm{K}_{\mathrm{s}}}{1+\mathrm{e}} \mathrm{d} \ln \left(\mathrm{s}_{0}+\mathrm{P}_{\mathrm{atm}}\right)$ with $\mathrm{P}_{0}=\mathrm{p}^{\prime}$ if reaching Loading/Collapse curve, or $\mathrm{s}_{0}=\mathrm{s}$ if reaching Suction Increase curve

where $\mathrm{P}_{0}{ }^{*}$ : equivalent consolidation pressure at saturation :

$\left.\frac{\mathrm{P}_{0}^{*}}{\mathrm{P}^{\mathrm{c}}}\right|^{\lambda(0)-\kappa(0)}=\frac{\mathrm{P}}{\mathrm{P}_{0}} \theta^{\lambda(\mathrm{s})-\kappa(\mathrm{s})}$

$\mathrm{P}^{\mathrm{c}}$ : reference pressure $=1 \mathrm{~atm}$,

$\lambda(\mathrm{s})=\lambda_{0}\left[(1-\mathrm{r}) \mathrm{e}^{-\beta \lambda \mathrm{s}}+\mathrm{r}\right](\mathrm{s}$ in $\mathrm{Pa})$

$\lambda_{0}=0.15 ; r=0.75 ; \beta_{\lambda}=0.05 \mathrm{~Pa}^{-1}, \lambda_{\mathrm{s}}=$ cste

Poisson's coefficient : $v=0.4$, shear modulus $G=3$ MPa.

According to the experimental protocol, the assumptions and the parameters, the samples should be initially (namely, after the initial compaction and release) over-consolidated by about $\mathrm{P}_{0}=8 \mathrm{MPa}$ for a suction $\mathrm{s}=350 \mathrm{MPa}$, which corresponds to a preconsolidation pressure at saturation $\mathrm{P}_{0} * 06 \mathrm{MPa}$. However, this initial compaction stage has not been experimentally surveyed, so that, since the mechanical parameters vary during density change, and are not known, a significant mis-estimation of the initial state is to be expected. In this frame, plastification should be unlikely to occur, as experimental swelling pressure will show never to exceed 2.5 MPa.

The sample parameters are : Sample height : 30 mm; Overall density (after initial compaction) : $\mathrm{d}=$ 1.6; Overall porosity : $\omega_{0}=40 \%$; Overall saturation : $\mathrm{S}_{0}=32 \%$

\subsection{Numerical results and sensitivity analysis}

The coupled equations have been solved numerically by means of the finite element code CASTEM2000 (Volckaert 2000). Results show that the hydromechanical process is fully coupled as expected. In the area subjected to imbibition, the suction decreases and, due to swelling, the isostatic (isotropic) pressure increases. During this process, both stiffness to pressure $\kappa$ and stiffness to suction $\kappa_{\mathrm{s}}$ increase. This explains why the swelling front moves upwards by compressing the upper levels, and letting the lower ones unchanged. This non-linear compression also explains the porosity profile: highered in the vicinity of the lower boundary, and lowered elsewhere. The upper levels being more and more impervious, as their porosity falls down under the compression by the lower levels, the imbibition process slowly decelerates. The importance of the coupling between hydraulics and mechanics is again visible on the water intake (fig. 6) : the complete saturation is achieved quicker if hydraulics are calculated without any mechanical inference, than if hydraulic parameters variation with mechanics are taken into account at each calculation step. 


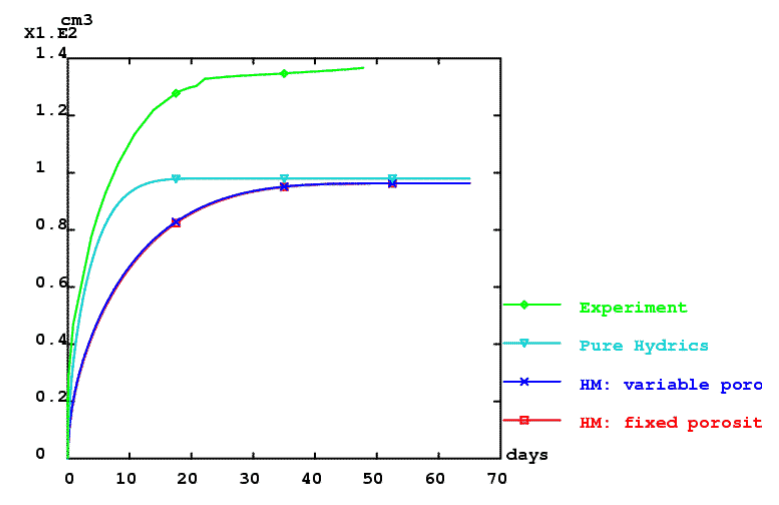

Water Intake

Figure 6. Total water absorbed by the sample. Experimental (diamond), constant porosity (inverse triangle) and coupled HM process (cross).

Using reference parameters leads to the following comparison between model and experience (fig. 7) : The swelling pressure is overestimated, $\mathrm{P}_{\mathrm{C} 0}=6 \mathrm{MPa}$, the saddle-like shape of swelling pressure versus time is not observed, the asymptotical state is achieved about twice later, the total water intake is under-estimated by $30 \%$. These poor results suggest that either the model, or the parameters, or both must be revised. We present in the following a sensitivity analysis to several parameters.

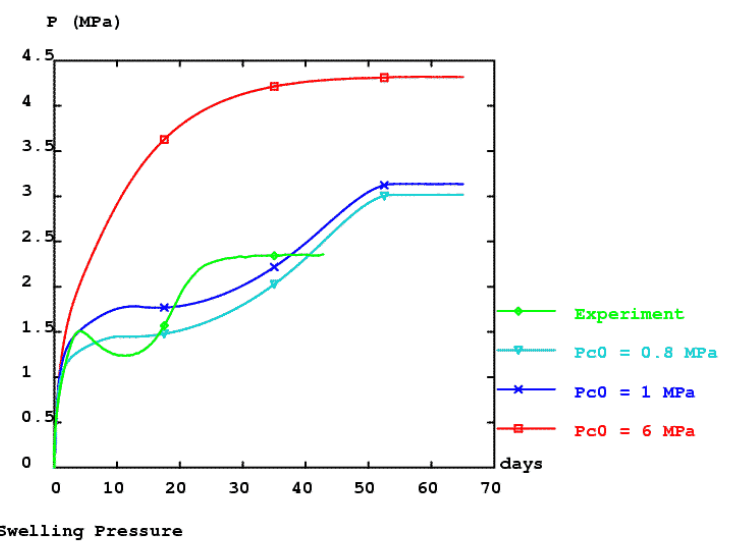

Figure 7. Swelling pressure versus time, using different preconsolidation levels $\mathrm{P}_{0}$ * . Reference value is $6 \mathrm{MPa}$.

Let us emphasize the importance of stiffness under pressure and suction changes, $\square$ and $\square_{s}$, and their variations with pressure and suction. The available $\square$ laws (Volckaert 2000) (linear or logarithmical) give completely different values according to the choice. Theoretically, this, along with an imprecise knowledge of $\square_{s}$, should have a high importance on the global mechanical response of the sample, since they control the elastic deformation of the medium (fig. 8).

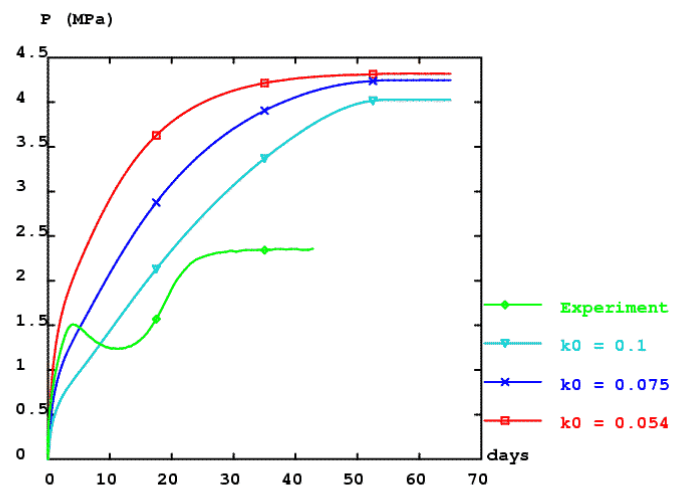

Influence of the saturated kapa k0 on the Swelling Pressure

Figure 8. Variation of the swelling pressure with saturated stiffness $\kappa_{0}$. Reference value is 0.054 .

When the pre-consolidation pressure $\mathrm{P}_{0} *$ decreases down to $0.8 \mathrm{MPa}$, a saddle-like shaped swelling pressure versus time slightly appears (fig. 7 ), suggesting clay plastification. Increasing the consolidation coefficient at saturation $\left(\square_{0}\right)$ - thus increasing plastification rate - lowers the swelling pressure and accentuates further the saddle shape. However, the corresponding $\mathrm{P}_{0} *=1 \mathrm{MPa}$ value seems inadequate. We are possibly facing another type of plasticity described by Gens (Volckaert 2000) consisting in some kind of "irreversible elastic deformations". Let us remind that Alonso (Alonso 1990) warned that his model was not suited to strongly swelling clays. The intrinsic permeability dependence with porosity has no observable impact (so it is not shown in this paper), but its absolute value should influence directly the global kinetics. A misestimating suction variation with saturation may also lead to misestimated kinetics.

The best fit was achieved for the following set of parameters (all others left unchanged) :

$\mathrm{P}_{0}{ }^{*}=0.1 \mathrm{MPa}$ instead of $6 \mathrm{MPa}$,

$\lambda_{0}=0.27$ instead of 0.15 ,

$\kappa_{0}=0.063$ instead of 0.054

$\mathrm{k}_{\mathrm{int}}=1.35 \cdot 10^{-20} \mathrm{~m}^{2}$ instead of $6.21 \cdot 10^{-21} \mathrm{~m}^{2}$

The swelling pressure results are quite satisfactory : the general kinetics is correct, the saddle-like shape is observed, though a little weaker, and the asymptotical value is correct (fig. 9). On the other hand, the water intake asymptotical value is still 30\% lower than experimentally observed (not represented because invariant with the modified parameters). 


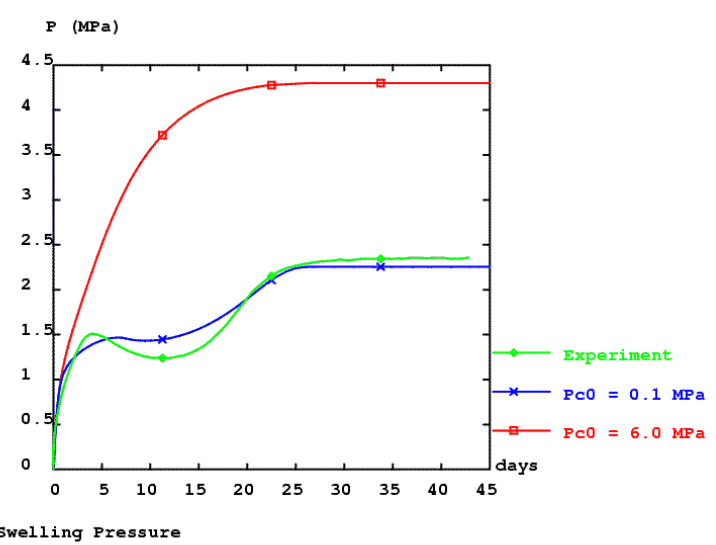

Figure 9. Swelling pressure obtained using roughly optimized parameters

\section{CONCLUSION}

During the imbibition process, plastification under pressure increase occurs. This should appear surprising if only overall preconsolidation pressure is taken into consideration. In fact, there are two preconsolidation pressures describing the respective state of clay powder and pellets. During compaction, the powder is very loose and densifies easily with a moderate consolidation, whereas the very dense pellets strongly resist, thus necessitating a high compaction pressure. Therefore, although a small amount of powder must be highly consolidated between the pellets, a mean preconsolidation pressure about $1 \mathrm{MPa}$, or even 1 atm (no consolidation) for this constituent seems reasonable. The observed plastification should then be the powder's only, so that the powder should undergo most of the swelling and compaction.

The hydro-mechanical imbibition process is strongly coupled and non-linear. The clay swells in the vicinity of the imbibition frontier, and compacts elsewhere.

As previously mentioned, the sample is heterogeneous, made of a mixture of powder and compacted pellets. The hydration is homogeneous, as confirmed by the modeling and the experimental observation. The up-scaling of the hydraulic process should thus be straight-forward.

On the other hand, pellets obviously constitute preferential paths to stresses, protecting the powder. They must pile up during the compaction, may break for some of them, but never plastify since the swelling pressure (2.5 $\mathrm{MPa}$ at best) never reach their preconsolidation pressure (at least $6 \mathrm{MPa}$ ). So that, roughly, the sample has the plastification of the powder, and the stiffness of the pellets. The homogenization (or up-scaling) of the mixture behavior to large scale borehole sealing seems delicate and uncertain. Some further studies will be necessary to understand how the mixture behavior can be deduced from characteristics of its components. From this point of view, we explain the saddle-like shape of the swelling pressure evolution through time by both plastification and heterogeneity.

The clay mechanical parameters were insufficiently known ; but their crude optimization allowed the code Castem2000 and the Alonso's model to reproduce satisfactorily the experimental swelling pressure. Unfortunately, the water intake proved independent on $\kappa_{0}, \alpha_{\kappa}, \lambda_{0}, \mathrm{P}_{0} *$. It is not clear yet which process controls the total porosity changes, that would explain the underestimation of total water absorption by the sample.

\section{REFERENCES}

Alonso E. E., Gens A., Josa A. 1990. A constitutive model for partially saturated soils. Geotechnique, vol 40, n³10, pp 405-430.

Dereeper B., Volckaert G., Imbert C., Villar M.V. 2001. Pellets/powder mixture of bentonite for backfill and sealing of HLW repositories. Clay Science for Engineering, Adashi \& FUKUE (eds). Balkema, Rotterdam, ISBN 905809 1759.

Pasquiou, A. 2001. Pellets d'argiles gonflantes. Elaboration et caractérisation hydromécanique. Thèse de doctorat présentée à l'Université des Sciences et Techniques de Lille.

Volckaert G. \& al. 2000. A large scale in situ demonstration test for repository sealing in an argillaceous host rock. RESEAL Project - Phase 1. European Commission Nuclear Science and Technology. Final Report EUR 19612 EN.

Volckaert G., Bernier F. and Dardaine M. 1996. BACCHUS 2, demonstration of the in situ application of an industrial clay based backfill material. European Commission Nuclear Science and Technology. Final Report EUR 16860.

\section{ACKNOLEDGEMENTS}

This study has been made with the support of the European Commission. 